\title{
Tales of methylomes and centrosomes in the human embryo
}

\author{
David F. Albertini ${ }^{1}$
}

Published online: 7 November 2015

(C) Springer Science+Business Media New York 2015

Nothing pleases patients more than the arrival of good news the day after ovum pickup. The "morning after" headliners brought to the attention of couples undergoing infertility treatment have as much to do with receiving the head count of fertilized eggs as it does with the anticipation of just how many will take the journey to producing a live birth. It comes as no surprise then that while the news of fertilization is uplifting, proof of the pudding awaits the outcomes of embryo culture.

The opening act staged deep within the embryology laboratory was long thought to hold the secret to success given the ability to recognize male and female pronuclei striving towards syngamy and that tell-tale sign of second polar body extrusion, the true sentinel of the end of meiosis. And beyond the patently visible, it is with confidence that observers of human embryology associate the onset of the earliest cleavage divisions with what must have been the timely execution of those initial cell cycles hand in hand with the duplication of paternal centrosomes introduced by fertilization or ICSI. The wonderful world of morphokinetics has taken to task the temporal cadence of those initial cell divisions with algorithm after algorithm being proffered as a useful standard for embryo selection to improve pregnancy chances. But, the lingering question is whether any of the currently used measures

Capsule Recent studies on pronuclear chromatin remodeling, in conjunction with tracking of centrosomes in human embryos, raise questions regarding the significance of pronuclear number as indicators of blastocyst and implantation potential.

David F. Albertini

DALBERTINI@kumc.edu

1 University of Kansas Medical Center, Kansas, KS, USA from time-lapse recordings report on the fidelity of egg activation and the earliest cell cycle-based events.

This month, a series of papers approach this question from different angles, offering our readership something to think about before investing in time-lapse technology as it presently stands. The first of these studies comes from Neyer and colleagues, who query the impact of paternal factors and sperm quality on several morphokinetic parameters based on the IMSI scoring method ("The impact of paternal factors on cleavage stage and blastocyst development analyzed by time-lapse imaging - a retrospective observational study" 10.1007/s10815-015-0558-3). Interestingly, while some of the early morphokinetic parameters examined displayed predictive value for blastocyst development, others were unable do so based on ICSI with sperm bearing structural line abnormalities. Studies of this kind reinforce what has been obvious when male factor is a consideration in developing a treatment plan, and they also raise the issue of launching embryogenesis with defective gametes that precludes neither achieving fertilization nor sustaining development in many cases to the blastocyst stage with transfer likely based on an apparently positive morphokinetic profile. For patients demonstrating repeated pregnancy failures with suspected male involvement, looking a bit deeper into the initial steps of fertilization would improve pregnancy chances.

Such is the message from the latest paper in JARG from the Mio laboratory in Japan (Kai et al., "Diagnosis of abnormal human fertilization status based on pronuclear origin and/or centrosome number" $10.1007 / \mathrm{s} 10815-015-0568-1)$. If you have not already done so, we encourage our readership to follow the work of Mio and colleagues as they have established a reputation for utilizing time-lapse imaging and high-resolution confocal microscopy to reveal the inner workings of the human embryo including their discovery of a fast 
block to polyspermy in humans. Practitioners of human ARTs have long been sensitized to the need to eliminate all embryos from consideration for eventual transfer if they contain less than or more than two pronuclei. And along with the shift from IVF to ICSI came the adjustment of original discriminators that would herald the origin of triploids not from polyspermy but from failed polar body extrusion. Characterizing the exact origins and composition of single pronuclear eggs and those bearing three pronuclei after either IVF or ICSI was the aim of this most recent study bringing a novel technological twist to the analysis that had yet to be employed in human ARTs.

Gender-specific demethylation pronuclear genomes is known to be one of the initial events in egg activation associated with chromatin remodeling and allows for the discrimination of male and female pronuclei based upon distinct methylation marks (see male pronucleus on the cover this month). While assays providing unequivocal results are few and far between, the one used by Kai and colleagues clearly distinguished pronuclei by gender in a range of single, double, or triple pronuclear embryos produced by either IVF or ICSI. The confirmation of male centrosome duplication in anticipation of syngamy in the same samples in which the parental origin of pronuclei could be established is encouraging, as is tracing the origins of tripolar spindles at the first cell cycle in cases of polyspermy. But what is most surprising was the result obtained in single pronuclear embryos harboring a correct diploid parental genome along with duplicated centrosomes, a result that could not possibly have been detected by traditional means. Besides offering a glimpse into the plasticity of the early embryo, this approach could be adopted in a clinical setting with patients prone to pronuclear abnormalities or early cell cycle defects that may not compromise the viability of resultant embryos.

The larger message deriving from studies like this is to urge caution and deliberation as new technologies enter the arena of human ARTs. Ideally, what we see encroaching upon the routine practice of embryo selection may offer benefits and improved pregnancy chances to some patients while indiscriminate application will only add to the emotional and financial burdens in patients for who embryo selection would be ill-advised.

Be on the lookout for our final issue of 2015 and the top ten discoveries in reproductive medicine that are likely to enter into the future parlance and practice of human ARTS. 